\title{
Simulation in Amorphous Silicon and Amorphous Silicon Carbide Pin Diodes
}

\author{
Dora Gonçalves $^{1}$, Miguel Fernandes ${ }^{1,2}$, Paula Louro ${ }^{1,2}$, Alessandro Fantoni ${ }^{1,2}$, \\ and Manuela Vieira ${ }^{1,2,3}$ \\ ${ }^{1}$ ADEETC, ISEL, Lisbon, Portugal \\ ${ }^{2}$ CTS-UNINOVA, Caparica, Portugal \\ ${ }^{3}$ DEE-FCT-UNL, Caparica, Portugal \\ \{dgoncalves, mfernandes, plouro, afantoni\} @deetc.isel.ipl.pt, \\ mv@isel.pt
}

\begin{abstract}
Photodiodes are devices used as image sensors, reactive to polychromatic light and subsequently color detecting, and they are also used in optical communication applications. To improve these devices performance it is essential to study and control their characteristics, in fact their capacitance and spectral and transient responses. This study considers two types of diodes, an amorphous silicon pin and an amorphous silicon carbide pin, whose major characteristics are simulated, using the AFORS-HET program . The pin diode structure can be defined using contacts, interfaces and optical layers and then common measurements can be simulated by a numerical model, the AFORSHET program. I-V, C-V characteristics, spectral response are simulated for both devices, without and under different illumination wavelengths. The results will allow a comparison between the main properties of amorphous silicon and amorphous silicon carbide diodes. We can conclude that sinusoidal frequency varies capacitance values as well as incident light wavelength. And when carbon is included in an amorphous silicon diode structure, its electrical and optical properties change.
\end{abstract}

Keywords: Photodiode, amorphous silicon, background illumination.

\section{Introduction}

This paper concerns the following research question: "By superimposing background illumination the device behaves as a filter, producing signal attenuation, or as an amplifier, producing signal gain, depending on wavelength combination. Is it possible (and if yes, how) to improve the frequency response of a a-Si:H photodiode for optoelectronic applications?", taking into consideration the next research hypothesis: "It is possible to improve the device performance by controlling the material properties (defect density, conductivity and light absorption) and layer thicknesses in order to get to an optimal definition of the device photo-capacitance".

Photodiodes are widely used for a variety of optoelectronic applications; one of the most important is their use in the context of optical communications. The state of 
the art of information transmission over optical fibers systems is well established over the Infra-Red technologies for light emitting, transmission and receiving, allowing communication with large (and always increasing) bandwidth characteristics. Nevertheless recent advances in the last two decades have produced an increasing market growth for solid state technology illumination with Light Emitting Diodes (LED), because it reduces considerably power consumption and expands architectural capabilities. Visible light wireless communication efforts, specifically with Light Emitting Diodes (LED), for indoor spaces have gained great interest lately and have already been included in standardization activities (IEEE 802.15) [1].

Amorphous Silicon pin structures, well studied during the 90's as solar cells devices for terrestrial photovoltaic applications and low power electronic systems [2], are getting a renewed interest as photodiodes devices working in the visible light range for communication applications [3]. The literature about the physics of a-Si:H devices is mainly centered on device characteristics and effects which are important for development and optimization of solar cell devices. Less attention has been paid to frequency response and, generally speaking, to the transient characteristics of the a$\mathrm{Si}: \mathrm{H}$ multilayer structures. The characterization of the device under pulsed light illumination has highlighted the presence of transient effects which are not measured under steady-state condition [4], [5]. At a first approach, these effects have been related to a photo-capacitive behavior of the a-Si:H structures, revealing a need for a more detailed analysis, able to relate the light controlled device capacitance to the physical characteristics of the device, like interface quality and/or defect density of the materials used. Our simulation program ASCA [6] has been found not able to reproduce the device transient characteristics under pulsed light conditions. This paper report our initial study based on simulation performed with the simulation program AFORS-HET [7]. We report here the simulation results about CurrentVoltage characteristics, Spectral Responses, and Capacitance Voltage characteristic. The simulations have been performed under different conditions of illumination and applied bias. Two different kind of photodiodes have been selected for our simulations: one pin structure of a-Si:H and one pin structure of a-SiC:H. The intent of this study is the comparison of the optoelectronic properties of this two kind of structures.

\section{Collective Awareness Platforms for Sustainability and Social Innovation}

Collective Awareness Platforms for Sustainability and Social Innovation (C.A.P.S.) are systems that combine data from real environments ("Internet of Things"), open online social media, and disseminated knowledge creation in order to generate new forms of social innovation by means of awareness of problems and possible solutions using collective efforts. The work hereby presented is motivated by a clear research question: Is it possible (and if yes, how) to improve the frequency response of a a$\mathrm{Si}: \mathrm{H}$ photodiode for WDM applications? The answer to this question is directly related to a future development of a network based on visible range optical communications. This technology will certainly contribute to the future development of C.A.P.S. 


\section{Simulation}

The AFORS-HET simulations have been performed under different conditions of illumination and applied bias. Two different kind of photodiodes have been selected one pin structure of a-Si:H and one pin structure of a-SiC:H. The intent of this study is the comparison of the optoelectronic properties for these two kinds of structures. Varying the i-layer thickness of the photodiodes we have simulated the I-V and C-V characteristics and the spectral response. The material parameters used in the simulator are reported in Table 1. Our study is mainly directed to study the influence of the intrinsic layer. The adding of carbon into the a-Si:H base is assumed to provoke a lager optical gap $(E g)$, an increasing of the defect density (Ntr) and a lowering of the carriers mobility $(\mu n, \mu p)$.

Table 1. Material parameters used in the AFORS-HET simulations (i-layer)

\begin{tabular}{|l|l|c|c|}
\hline Parameter & Description & a-Si:H & a-SiC:H \\
\hline $\mathrm{E}_{\mathrm{g}}(\mathrm{eV})$ & Energy gap & 1.72 & 2.1 \\
\hline$\mu_{\mathrm{n}}\left(\mathrm{cm}^{2} \mathrm{~V}^{-1} \mathrm{~s}^{-1}\right)$ & Electrons mobility & 20 & 2 \\
\hline$\mu_{\mathrm{p}}\left(\mathrm{cm}^{2} \mathrm{~V}^{-1} \mathrm{~s}^{-1}\right)$ & Holes mobility & 5 & 0.5 \\
\hline $\mathrm{E}_{\mathrm{U}, \mathrm{v}}(\mathrm{eV})$ & Urbach energy (valence tail) & 0.05 & 0.05 \\
\hline $\mathrm{E}_{\mathrm{U}, \mathrm{c}}(\mathrm{eV})$ & Urbach energy (conduction tail) & 0.035 & 0.035 \\
\hline $\mathrm{N}_{\mathrm{tr}}\left(\mathrm{cm}^{-3}\right)$ & Defects density & $5 \times 10^{15}$ & $5 \times 10^{16}$ \\
\hline $\mathrm{N}_{\mathrm{tr}}\left(\mathrm{cm}^{-3}\right)$ & Defects density & $5 \times 10^{15}$ & $5 \times 10^{16}$ \\
\hline
\end{tabular}

In Fig. 1 and 2 are reported, respectively, the short circuit current and the open circuit voltage produced by a $\mathrm{Si}: \mathrm{H}$ pin structure as a function of the i-layer thickness. The simulation has been performed under illumination with different wavelength: red $(650 \mathrm{~nm})$, green $(560 \mathrm{~nm})$, blue $(440 \mathrm{~nm})$ and violet $(410 \mathrm{~nm})$. The intrinsic layer thickness varies between 200 and $1100 \mathrm{~nm}$. The blue radiation is absorbed in the front part of the device, so an increasing of the i-layer thickness produces a bottom region subject to a low illumination level. The series resistance of the photodiode increases and the overall photocurrent falls down. When illuminated with a red light the increasing of the i-layer thickness produces an enhancement of the photocurrent. This is due to the lower absorption coefficient in the red part of the spectrum and the higher penetration of the red radiation within the pin structure. With a value of 1100 $\mathrm{nm}$ for the i-layer thickness, the short circuit current saturates. A further increasing of the i-layer thickness would not produce any enhancement and it would end in a photo current degradation. A thickness of $400 \mathrm{~nm}$ appears to be an optimal choice for absorption of a green radiation. This would be the optimal choice for a solar cell device. For all the radiations, the open circuit voltage suffers a smooth lowering with the i-layer thickness. As matter of fact a photodiode for communication application would not work in photovoltaic mode, but will be always reversed biased. The photocurrent is the most important parameter to be extracted by these simulation runs. 


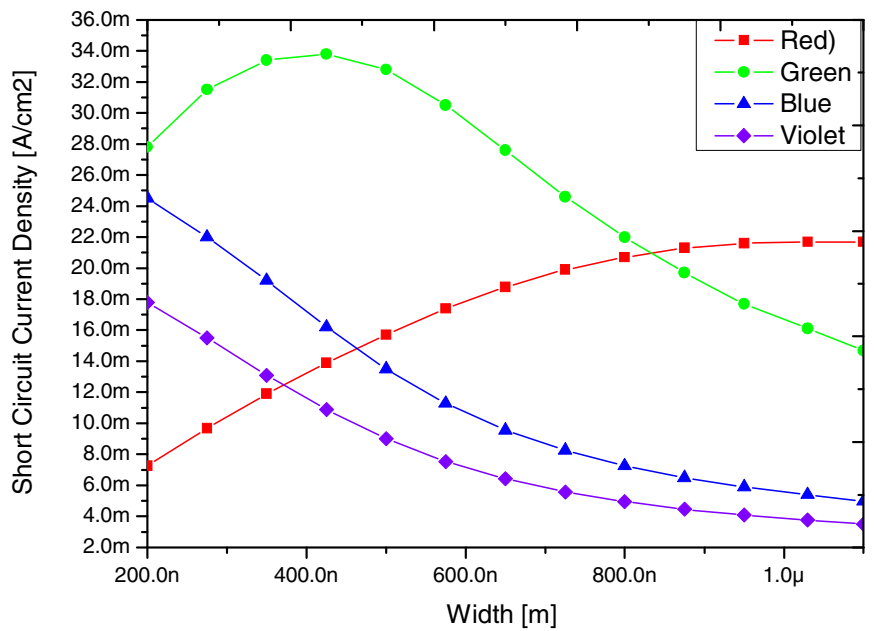

Fig. 1. Short circuit current produced under different illumination conditions by a a-Si:H pin structure as a function of the i-layer thickness

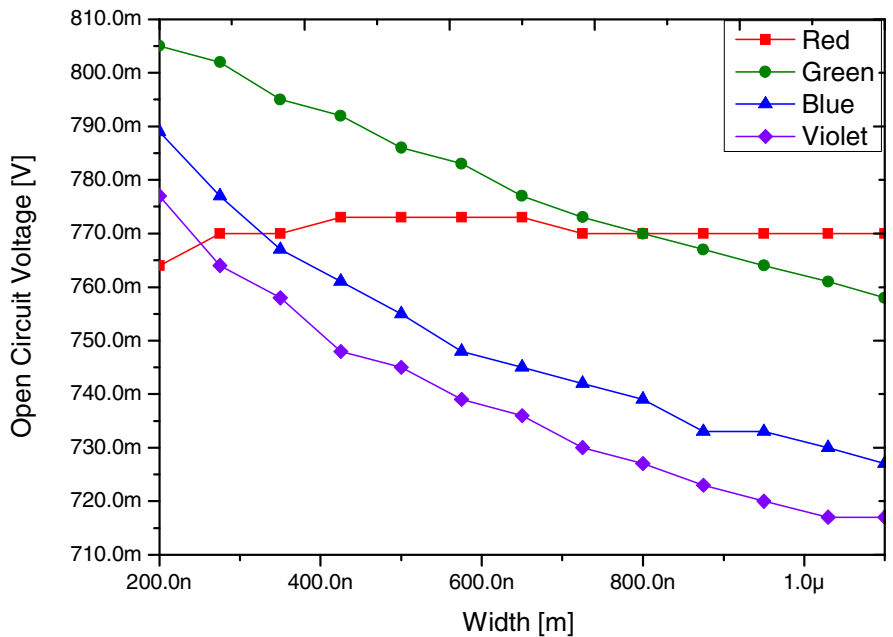

Fig. 2. Open Circuit Voltage produced under different illumination conditions by a a-Si:H pin structure as a function of the i-layer thickness

In Fig. 3 is reported the short circuit current produced by a a-SiC:H pin structure as a function of the i-layer thickness. Like in the precedent case, the simulation has been performed under illumination with different wavelength: red $(650 \mathrm{~nm})$, green $(560 \mathrm{~nm})$, blue $(440 \mathrm{~nm})$ and violet $(410 \mathrm{~nm})$. Again, the intrinsic layer thickness varies between 200 and $1100 \mathrm{~nm}$. The larger optical gap produces a very low absorption in the red range and the photocurrent under red illumination is almost zero. The photodiode has a good response in the blue spectrum, while under green irradiation 


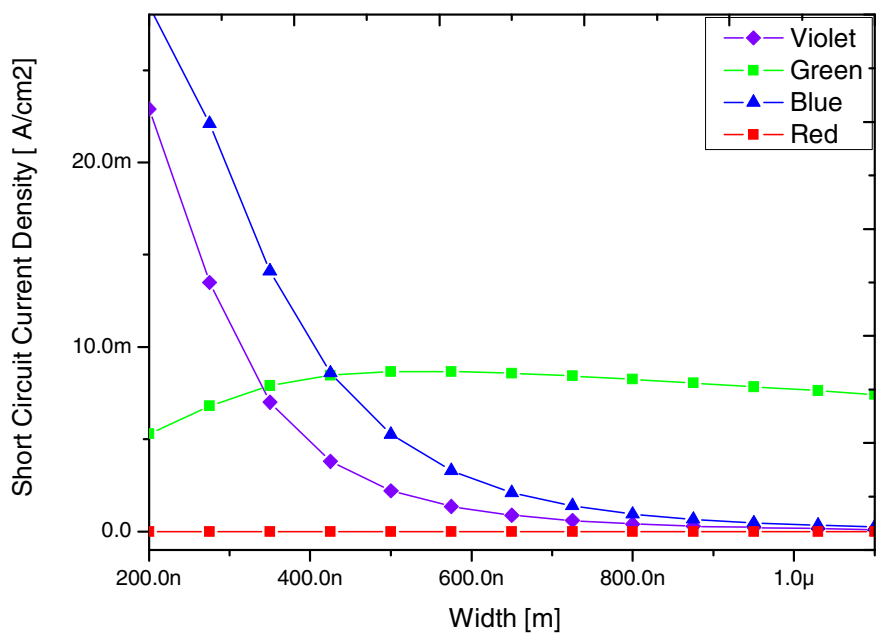

Fig. 3. Short circuit current produced under different illumination conditions by a a-SiC:H pin structure as a function of the i-layer thickness

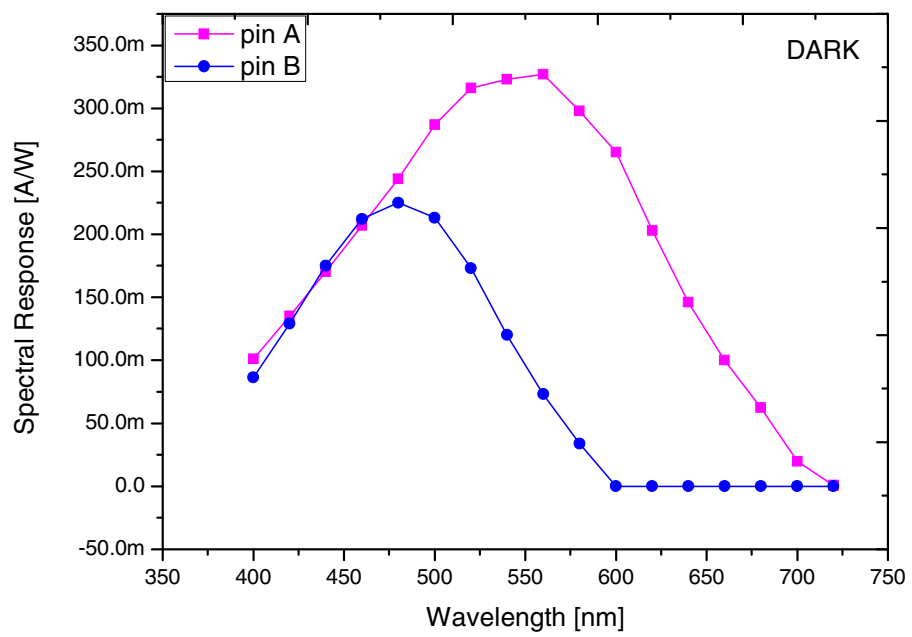

Fig. 4. Spectral response of a a-Si:H (Pin A) and a a-SiC:H (pin B) structure. The i-layer thickness is $500 \mathrm{~nm}$ for both cases.

the photocurrent is a lot lower than in the a-Si:H case. This is caused by the inferior value of the free carrier mobility. The increasing of the i-layer thickness produces a fast degradation of the photocurrent. This is the effect of the augmented density of defects and the consequentially increased trapped charge density and recombination centers. A similar behavior was already reported for a-Si:H pin solar cell with increasing density of defect in the intrinsic layer [8].

In Fig. 4 is depicted the spectral response of a a-Si:H and a a-SiC:H structure. The i-layer thickness is $500 \mathrm{~nm}$ for both cases. In both structures the curves exhibit a 
similar trend. The spectral sensitivity increases with the wavelength until reaching a maximum, and then exhibits a decrease reaching very low values. It is also observed that for the a-SiC:H structure the sensitivity is confined to the range $400-600 \mathrm{~nm}$, while the a-Si:H device exhibits sensitivity in the whole visible range. This difference is related mainly to the energy gap of both materials. It is also observed that the maximum of each curve occurs at different wavelengths depending on the nature of the material. For the a-Si:H photodiode it is located around $560 \mathrm{~nm}$ while for the a$\mathrm{SiC}: \mathrm{H}$ device at $480 \mathrm{~nm}$. This is related mainly to the energy gap and absorption coefficients of each absorber layer. For the a-Si:H structure the magnitude of the spectral response is higher than the observed for the a-SiC:H structure, except in the short wavelength range (up to $470 \mathrm{~nm}$ ) where the curves overlap.

In Fig. 5 it is displayed, respectively, the capacitance-voltage characteristic (under reverse bias) for the device a-SiC: $\mathrm{H}$ at different illumination conditions (dark, red, green, blue and violet light). The $\mathrm{C}-\mathrm{V}$ measurement is simulated by considering an applied AC voltage of $0.02 \mathrm{~V}$ with frequency of $1 \mathrm{kHz}$. It is observed that under red and green light the capacitance remains low and it is independent on the applied bias, while under blue and violet light it decreases with the increase of the reverse bias. Maximum values are observed with violet illumination at short circuit voltage (230 $\left.\mathrm{nF} / \mathrm{cm}^{2}\right)$.

In Fig. 6 it is displayed the $\mathrm{C}-\mathrm{V}$ characteristic of the structure a-SiC:H under violet illumination simulated at different $\mathrm{AC}$ frequencies in the range $1 \mathrm{kHz}$ up to $100 \mathrm{kHz}$. Results show that the capacitance trend with the reverse bias is identical for every frequency. Higher values of capacitance correspond to low frequency values $(1 \mathrm{kHz})$.

Practical measurements are shown in Fig. 7, for comparison.

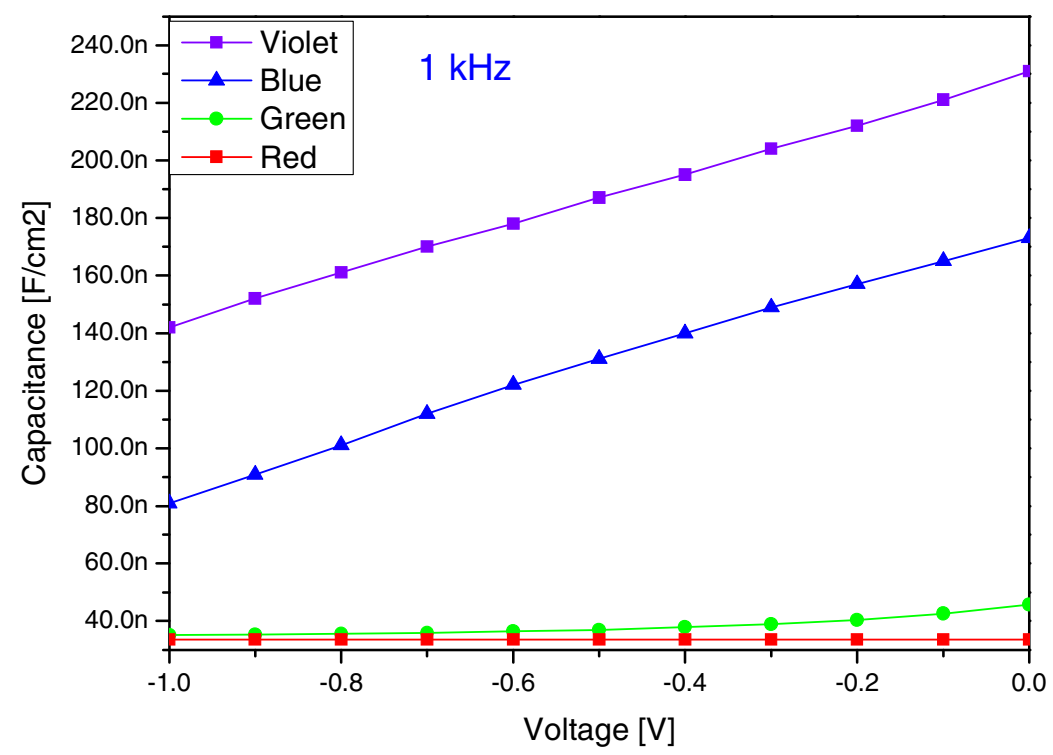

Fig. 5. C-V characteristic of the a-SiC:H structure at $1 \mathrm{kHz}$ under different illumination conditions 


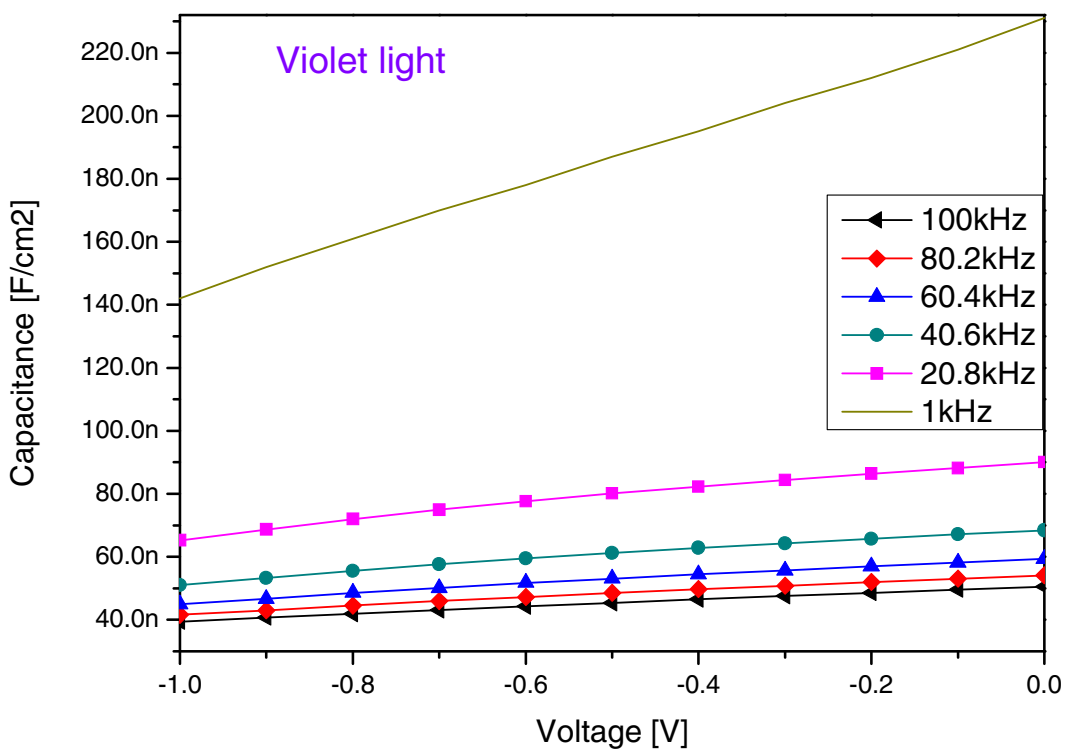

Fig. 6. C-V characteristic of the a-SiC:H structure under violet light simulated at different AC frequencies

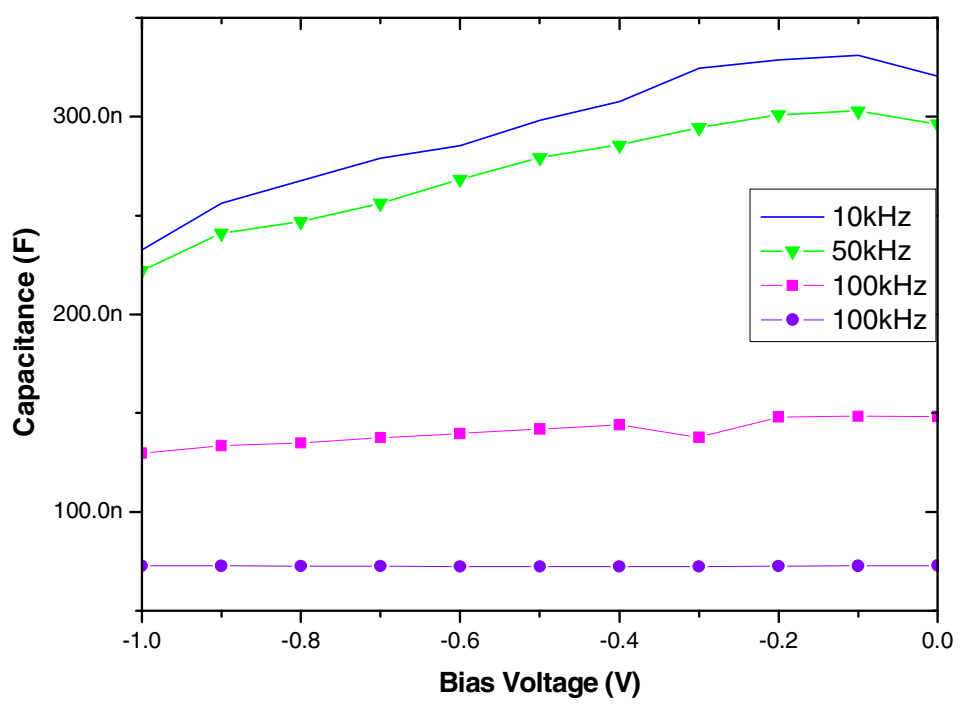

Fig. 7. C-V diagram for an amorphous silicon pin diode, under dark condition (practical measurements) 


\section{Conclusions and Future Work}

We have performed a simulation study on the electrical characteristics of an a-Si:H pin photodiode and compared to a a-SiC:H pin photodiode.

I-V characteristic, for an amorphous silicon sample, shows that short circuit density current has a maximum for a pin intrinsic layer width around $400 \mathrm{~nm}$, when illuminated with green light. When using blue or violet illumination, values of short circuit current reduce with thickness, although when using red illumination it increases. $\mathrm{C}-\mathrm{V}$ characteristic depends on frequency and illumination, the peak values corresponding to $1 \mathrm{kHz}$ and violet light.

For a better understanding of these characteristics the transient response under different wavelength pulsed light must to be simulated and related to capacitive effects. The AFORS-HET simulator is very powerful simulation software when applied to pin structures, but it does not allow for simulation of multi-junction devices. Our focus in future work will be on the development of a transient model able to simulate the photo capacitive effects in a-Si:H/a-SiC:H double junction photodiodes.

\section{References}

1. Rajagopal, S., Roberts, R.D., Lim, S.-K.: IEEE 802.15.7 visible light communication: modulation schemes and dimming support. IEEE Communic. Magazine 50 (2010)

2. Deng, X., Schiff, E.A.: Amorphous Silicon-based Solar Cells. In: Handbook of Photovoltaic Science and Engineering. John Wiley \& Sons (2003)

3. Vieira, M., Vieira, M.A., Louro, P., Fernandes, M., Fantoni, A., Silva, V.: SiC multilayer photonic structures with self optical bias amplification. In: MRS Proceedings, vol. 1426 (2012)

4. Fantoni, A., Fernandes, M., Louro, P., Vieira, M.A., Vieira, M.: Capacitive effects in pinpin photodiodes. Microelectronic Engineering 108, 195-199 (2013)

5. Gonçalves, D., Fernandes, L.M., Louro, P., Vieira, M., Fantoni, A.: Measurement of Photo Capacitance in Amorphous Silicon Photodiodes. In: Camarinha-Matos, L.M., Tomic, S., Graça, P. (eds.) DoCEIS 2013. IFIP AICT, vol. 394, pp. 547-554. Springer, Heidelberg (2013)

6. Fantoni, A., Vieira, M., Cruz, J., Schwarz, R., Martins, R.: A two-dimensional numerical simulation of a non-uniformly illuminated amorphous silicon solar cell. Journal of Physics D: Applied Physics 29, 3154 (1996)

7. Stangl, R., Leendertz, C., Haschke, J.: Numerical simulation of solar cells and solar cell characterization methods: the open-source on demand program AFORS-HET. In: Rugescu, R.D. (ed.) Solar Energy, pp. 319-352. INTECH (2010)

8. Fantoni, A., Viera, M., Martins, R.: Influence of the intrinsic layer characteristics on a-Si: H $\mathrm{p}-\mathrm{i}-\mathrm{n}$ solar cell performance analysed by means of a computer simulation. Solar Energy Materials and Solar Cells 73, 151-162 (2002) 